\title{
Fundamentals of Robotic Fabrication in Architecture: Multi Materiality, Multiscale Resolution and Multimode Production
}

\section{Sina Mostafavi ${ }^{1}$, Mohammad Saad Moharram ${ }^{2}$, Adeeb Khaeez ${ }^{3}$}

${ }^{1}$ TU Delft \& Dessau Institute of Architecture, Netherlands

${ }^{2,3}$ Dessau Institute of Architecture, Germany

${ }^{1}$ s.mostafavi@tudelft.nl

${ }^{2}$ mohamed.s.h.s.moharram@student.hs-anhalt.de

33Adib.khaeez@student.hs-anhalt.de

\begin{abstract}
This paper discusses the recent advancement in architectural materialization processes. The focus is on design to robotic production systems through which the realization of more efficient building processes and building products is achieved. Introducing three prototypical case studies, the research specifically addresses some of the major fundamentals of robotic production in architecture. Considering the fact, that building systems have consisted of numerous subsystems each with varying requirements, hybridity or multi-materiality is essential in architectural design. Therefore, computation to production of efficient hybrid systems with multiple materials is tested and prototyped in a series of case studies. The introduced multilateral system is a hybrid of concrete as the structural material and expanded polystyrene as the second substance. Beyond the description about computational design, digital modeling and robotic production methods, this project highlights how through using robotic production the process of mold making and concrete casting is re-examined. The second topic is the resolution, which is about simultaneous design to production in multiple scales, ranging from micro to macro. This is elaborated and prototyped in a case study on incremental metal forming. In this project, the very micro scale manipulation of bendable thin sheets of metal results in stiffness according to the design requirements. The last subject is explained in design to robotic production of a customized table with numerous components in expanded polystyrene that will be coated in fiberglass. In this design, multimode robotic production methods are implemented according the curvature analysis. As a result, the evaluation of the efficiency of the production process is feedback to design process in which hot wire cutting is applied to single curvature surfaces, while robotic milling is only applied where needed. Providing more details about the projects, the paper will conclude with a framework on fundamentals of robotic production in architecture.
\end{abstract}

Keywords: Architectural robotics; Multi materiality; Hybridity; Multiscale resolution; Multimode production; Incremental metal forming 